\title{
Induction of apoptosis by evodiamine involves both activation of mitotic arrest and mitotic slippage
}

\author{
LI-HONG ZHU ${ }^{1,3}$, WEI BI ${ }^{2}$, XIAO-DONG LIU ${ }^{3}$, JIE-FEN LI $^{3}$, \\ YING-YA WU ${ }^{3}$, BIAO-YAN DU ${ }^{3}$ and YU-HUI TAN ${ }^{3}$ \\ ${ }^{1}$ Department of Pathophysiology, Medical College of Jinan University, Guangzhou 510632; ${ }^{2}$ Department of Neurology, \\ First Affiliated Hospital of Jinan University, Guangzhou 510630; ${ }^{3}$ College of Fundamental Medical \\ Science, Guangzhou University of Chinese Medicine, Guangzhou 510405, P.R. China
}

Received June 2, 2011; Accepted July 7, 2011

DOI: $10.3892 /$ or.2011.1444

\begin{abstract}
Evodiamine (Evo) is an indole quinazoline alkaloid isolated from the fruit of Evodia rutaecarpa Bentham. Previous studies have shown that Evo exhibits anti-proliferative anti-tumor activities in several cancer types, but its target(s) and underlying mechanism(s) of action remain unclear. In the present study, we sought to establish a cell synchronization model in order to examine the anti-proliferative and apoptotic mechanisms of Evo in the human gastric cancer cell line SGC-7901. In addition, we transfected these cells with full-length or non-degradable (ND) cyclinB1 to evaluate the relationship between the induction of apoptosis and activation of mitotic arrest and mitotic slippage by Evo. Our results demonstrated that Evo markedly inhibited cell growth and was cytotoxic to SGC-7901 cells. Furthermore, transient Evo treatment $(<16 \mathrm{~h})$ caused reversible mitotic arrest, but sustained mitotic arrest was required to initiate apoptosis. The time required to reverse the apoptotic effects of Evo was between 16 and $20 \mathrm{~h}$. We also demonstrated that promotion of mitotic slippage by a CDK1 inhibitor enhanced apoptosis. Furthermore, we evaluated the effect of delaying mitotic slippage by overexpressing ND cyclinB1, which delayed apoptosis. In conclusion, these results indicate that Evo-induced apoptosis is associated with mitotic arrest and subsequent mitotic slippage, which may underlie the actions of Evo in the treatment and prevention of cancer.
\end{abstract}

Correspondence to: Dr Yu-Hui Tan, College of Fundamental Medical Science, Guangzhou University of Chinese Medicine, no. 12 Jichang Road, Sanyuanli, Guangzhou 510405, P.R. China

E-mail: tyuhui66@yahoo.cn

Abbreviations: APC, anaphase-promoting complex; Evo, evodiamine; ND, non-degradable; SAC, spindle assembly checkpoint; TD, thymidine

Key words: evodiamine, apoptosis, mitotic arrest, mitotic slippage, cyclinB1

\section{Introduction}

Evodiamine (Evo) is one of the principle bioactive compounds isolated from the dry, unripened fruit of Evodia rutaecarpa Bentham (known in Chinese as ' $w u$-chu-yu'). This natural compound affects many physiological processes, including gastrointestinal tract function (1), vasorelaxation (2) and exhibits cardiotonic effects (3) and has been used as astringent, analgesic, anti-emetic and anti-inflammatory agent and in the treatment of hypertension (4).

Recently, Evo has been reported to possess anti-cancer activities, including anti-proliferative (5-9), anti-invasive and anti-angiogenic effects (10). The anti-proliferative effects of Evo appear to be mediated via arresting the cell cycle (11). Specifically, Evo has been shown to affect the $M$ phase of the cell cycle in several tumor cell types $(12,13)$. This mitotic arrest results in the inhibition of cell proliferation and causes subsequent apoptosis of these Evo treated cells. However, the molecular targets of Evo and the mechanisms of action underlying its anti-proliferative and apoptotic effects are poorly understood. In general, tumors are characterized by a disrupted cell cycle and most cancer cells are able to progress through the cell cycle even in the absence of normal mitogenic signals $(14,15)$. Numerous compounds targeting the mitotic spindle have been used as cancer therapeutics. These include naturally occurring molecules such as taxanes and vinca alkaloids, which are regarded as 'mitotic spindle poisons' that interfere with microtubule dynamics by targeting tubulin at multiple sites (16-18). Cells exposed to these inhibitors become arrested in mitosis, which underlies the anti-proliferative activity of these drugs. Subsequently, blocked cells may remain arrested or enter interphase without chromosome segregation or cell division, a process known as mitotic slippage, which eventually leads to apoptosis. Thus, agents targeting the mitotic spindle of cancer cells undergoing cell cycle represent a specific therapeutic strategy for the treatment of human neoplasms (19-21).

The ability of Evo to induce apoptosis in cancer cells indicates that Evo may share a common mechanism with other therapies that can sensitize or induce the death of cancer cells (22) and thus, provides a strong rationale for its clinical use as an anti-tumor drug. However, the question remains as to 
whether the molecular events leading to Evo-induced apoptosis of cancer cells involve cell cycle control.

In the present study, we developed a cell synchronization model to examine the anti-proliferative and apoptotic mechanisms of Evo in SGC-7901 human gastric carcinoma cells. Furthermore, we transfected SGC-7901 cells with full-length (FL) or non-degradable (ND) cyclinB1 to examine the relationship between the induction of apoptosis and the activation of mitotic arrest and mitotic slippage mediated by Evo. Our results indicated that prolonged treatment of these cancer cells lead to irreversible cell death that involved mitotic slippage and the loss of cell cycle control in a cyclin-dependent kinase 1 and cyclinB1-dependent manner.

\section{Materials and methods}

Cell culture and drug treatment. SGC-7901 cells, from a human gastric carcinoma, were obtained from the Sun Yat-sen University Cell Collection and cultured in RPMI-1640 medium supplemented with 10\% FBS, 2 mM L-glutamine, $100 \mathrm{kU} / \mathrm{l}$ penicillin and $100 \mathrm{mg} / \mathrm{l}$ streptomycin (Gibco, Carlsbad, CA) at $37^{\circ} \mathrm{C}$ in $5 \% \mathrm{CO}_{2}$. Only cells in the exponential phase of growth were used for subsequent experiments.

Evo ( $98 \%$ pure as determined by HPLC) was obtained from the National Institute for the Control of Biological Products. Evo was dissolved in dimethylsulfoxide (DMSO) for the stock solution and was diluted in RPMI-1640 before experimental use. Cells were treated with vehicle (0.1\% DMSO) or Evo for various times and were then harvested for analysis.

Cell synchronization. Cell synchronization was carried out as described previously (23) with some modifications. Briefly, cells were treated with $2 \mathrm{mM}$ thymidine (TD) for $16 \mathrm{~h}$, followed by incubation in TD-free medium for $10 \mathrm{~h}$ and then treated again with $2 \mathrm{mM}$ TD for $16 \mathrm{~h}$. Cell synchronization at the G0/G1 boundary was confirmed by fluorescence-activated cell sorting (FACS). Blocked cells were released by incubation in TD-free medium containing 10\% FBS after three washes with phosphate-buffered saline (PBS).

Vector construction. CyclinB1 $\Delta 157$ (ND cyclinB1) with amino acid residues 1-157 removed and full-length cyclinB1 (FL cyclinB1) were amplified from a human cDNA library with primers (sense: 5'-CCGCTCGAGATGGCAGAAGATG GAGCTGATCC-3' for ND and 5'-CCGCTCGAGTGAGCTG CCTGGTGAAG-3' for FL; antisense: 5'-CGGGATCCAGAT ACACCTTTGCCACAGCCT-3' for both). The generated $830 \mathrm{bp}$ and $1301 \mathrm{bp}$ DNA fragments were subcloned into the pEGFP-N1 vector (Clontech, Palo Alto, CA) to construct pEGFP-N1-ND cyclinB1 and pEGFP-N1-FL cyclinB1 after digestion with XhoI and BamHI.

Flow cytometry. Adherent and floating cells were collected and fixed with $70 \%$ ethanol. Cells were then stained with $70 \mu \mathrm{g}$ / $\mathrm{ml}$ propidium iodide (PI) for $20 \mathrm{~min}$ at $37^{\circ} \mathrm{C}$ and analyzed by FACS.

Cells were synchronized at the G0/G1 boundary by double TD block. For continuous drug exposure experiments, after synchronization, cells were treated with vehicle (0.1\% DMSO) or $1 \mu \mathrm{M}$ Evo for $12,16,20,24,28,32$ or $36 \mathrm{~h}$ and then harvested for analysis. For drug washout experiments, cells were treated with vehicle or Evo for the various durations described above, and were then incubated for a further $12 \mathrm{~h}$ in the absence of drug before harvesting.

Upon release from synchronization $(0 \mathrm{~h})$, cells were treated with $7.5 \mu \mathrm{M}$ cyclin-dependent kinase 1 inhibitor (CDK1I), $0.5 \mu \mathrm{M}$ Evo or $1.0 \mu \mathrm{M}$ Evo for $24 \mathrm{~h}$ or with $0.5 \mu \mathrm{M}$ Evo or $1.0 \mu \mathrm{M}$ Evo for $18 \mathrm{~h}$ which was followed by the addition of $7.5 \mu \mathrm{M}$ CDK1I and incubation for a further $6 \mathrm{~h}$.

Cells were transfected with pEGFP-N1, pEGFP-N1-ND or pEGFP-N1-FL cyclinB1 using DNA-Lipofectamine 2000 (Invitrogen, Carlsbad, CA). After synchronization, cells were treated with vehicle or $1.0 \mu \mathrm{M}$ Evo for $36 \mathrm{~h}$ before FACS analysis.

Western blot analysis. Cells were synchronized as described above and then treated with $1 \mu \mathrm{M}$ Evo for $16,20,24$ or $36 \mathrm{~h}$. Both adherent and floating cells were collected. Western blotting was carried out as described previously (24) with some modifications. Briefly, cell pellets were lysed in RIPA buffer $(50 \mathrm{mM}$ Tris-HCl, pH 7.4, 1\% IGEPAL, 1\% sodium deoxycholate and $1 \mathrm{mM}$ PMSF). Protein levels in these cell lysates were quantified by the Bradford assay, separated by SDS polyacrylamide gel electrophoresis and transferred to a PVDF membrane for immunoblotting. Primary antibodies to cyclinB1 were obtained from Santa Cruz Biotechnology (Santa Cruz, CA,1:500) and primary antibodies to cyclinE and $\beta$-actin were obtained from Upstate Cell Signaling (Waltham, MA, 1:1000). The secondary antibodies (Upstate Cell Signaling) used were goat anti-rabbit $\operatorname{IgG}(1: 5000)$ and goat anti-mouse IgG (1:5000) conjugated with horseradish peroxidase (HRP).

Colony survival assay. Cells were seeded at $2 \times 10^{3} /$ well in 6-well plates and grown overnight. Cultures were subjected to the cell synchronization protocol as described above and then treated with $7.5 \mu \mathrm{M}$ CDK1I, $0.5 \mu \mathrm{M}$ Evo or $1.0 \mu \mathrm{M}$ Evo for $24 \mathrm{~h}$ or with $0.5 \mu \mathrm{M}$ or $1.0 \mu \mathrm{M}$ Evo for $18 \mathrm{~h}$, which was followed by the addition of $7.5 \mu \mathrm{M}$ CDK1I and a further incubation of $6 \mathrm{~h}$. After washout, cells were allowed to grow in drug-free medium for 7 days. Afterwards, cells were fixed with methanol:acetic acid (3:1) for $15 \mathrm{~min}$ and Giemsa-stained for $15 \mathrm{~min}$ at room temperature. Photographs were taken after extensive washing and colonies consisting of at least 50 cells were scored.

Fluorescence measurements of transfected cells. Two sets of transfections with each of the three constructs (pEGFP-N1, pEGFP-N1-ND cyclinB1 and pEGFP-N1-FL cyclinB1) were performed using DNA-Lipofectamine 2000. A total of $24 \mathrm{~h}$ after transfection, 1.0 $\mu \mathrm{M}$ Evo was added to one set (Evo group) and vehicle was added to the other set (control group). Thirty-six hours later, cells were examined under a fluorescent microscope for green fluorescence. Ten images for each treatment were taken from randomly chosen fields and scored by an observer who was unaware of the transfection vectors according to the brightness of the fluorescence with the following values: 1 point, $+; 2$ points, $++; 3$ points, +++ ; or 4 points, ++++ .

Statistical analyses. Continuous data are described with means and standard deviations and were compared with a 
t-test or one-way ANOVA. Categorical data are presented as frequencies and were compared using a Fisher's exact test. Data were analyzed using the SAS software (ver. 9.0; SAS Institute Inc., Cary, NC) and plotted using SPSS (ver. 15.0; SPSS, Inc., Chicago, IL). P-values $<0.05$ indicate statistical significance.

\section{Results}

Cell synchronization. To induce SGC-7901 cell cycle synchronization, we conducted a TD double blocking protocol as well as serum starvation. Serum starvation did not achieve an ideal synchronization state (data not shown). In contrast, TD double blocking maintained cells in their G0/G1 phase irrespective of dosage, thereby demonstrating effective synchronization of this gastric carcinoma cell line. Thus, we used a $2-\mathrm{mM}$ TD double block to induce cell cycle synchronization of SGC-7901 cells for this study and found that G0/G1-phase cells accounted for $77.3 \%$ of the cell population, while G2/M phase cells accounted for only $2.0 \%$ of total cells. After incubation for a further $12 \mathrm{~h}$ in TD-free medium, the proportion of cells in each phase returned to the normal range (Fig. 1A). These results show that the TD double block is effective for preparing a large number of cells in the G0/G1 phase.

Evo affects the cell cycle and induces apoptosis. The cell cycle distribution and apoptosis index were assessed by FACS after release from block followed by treatment with $1 \mu \mathrm{M}$ Evo for the various durations indicated (Fig. 1B and C). Evo treatment for $12 \mathrm{~h}$ caused the accumulation of cells in the G2/M phase, which was accompanied by a significant decrease in cell populations in both the $\mathrm{G} 1$ and $\mathrm{S}$ phases. Most cells $(78.8 \pm 2.6 \%)$ were arrested at $\mathrm{G} 2 / \mathrm{M}$, thereby containing $4 \mathrm{~N}$ DNA with few $(3.6 \pm 0.5 \%)$, if any, cells in sub-G1, which is indicative of apoptosis. After 16 h of treatment, cells arrested at G2/M increased to $90.7 \pm 4.1 \%$, with relatively few cells found in the apoptotic state $(4.1 \pm 1.1 \%)$. With a 12 - or 16 -h exposure followed by a further incubation for $12 \mathrm{~h}$ in drug-free medium (12R and $16 \mathrm{R}$, respectively), most of the arrested cells re-entered the cell cycle with few apoptotic cells observed. With prolonged drug treatment to $20 \mathrm{~h}, \mathrm{G} 2 / \mathrm{M}$ phase cells dropped to $73.5 \pm 2.9 \%$, but sub-G1 cells then accounted for $16.0 \pm 1.5 \%$. However, $40.4 \pm 2.0 \%$ of apoptotic cells appeared following incubation for an additional $12 \mathrm{~h}$ in the absence of Evo (20R). Evo exposure for $24,28,32$ or $36 \mathrm{~h}$ with a further $12 \mathrm{~h}$ incubation in drug-free medium reduced the $\mathrm{G} 2 / \mathrm{M}$ population and the sub-G1 cells were $34.0 \pm 2.9 \%, 32.9 \pm 3.1 \%, 57.3 \pm 3.8 \%$ and $52.5 \pm 2.6 \%$ (24R, 28R, 32R and 36R), respectively, thereby demonstrating a significant proportion of these cells were undergoing apoptosis (Fig. 1D). Together, these data suggest that transient Evo exposure caused a reversible mitotic arrest while sustained mitotic arrest was required for the initiation of apoptosis. These results indicate that the reversible time for Evo-induced apoptosis was 16-20 h. Importantly, cells cultured up to $36 \mathrm{~h}$ without Evo exposure exhibited normal cell cycling without significant apoptosis $(3.63 \pm 0.35 \%$, data not shown).

We next assessed whether a CDK1I could accelerate mitotic slippage and enhance apoptosis. Mitotic arrest and apoptosis were monitored by FACS analysis in order to assess DNA content. On release from block, exposure to $7.5 \mu \mathrm{M}$
CDK1I for $24 \mathrm{~h}$ resulted in $7.6 \%$ of cells in apoptosis (Fig. 2A, lower left panel), which was not significantly different from the control (Fig. 2A, upper left panel). Additionally, $0.5 \mu \mathrm{M}$ Evo for $24 \mathrm{~h}$ with co-incubation of CDK1I for the last $6 \mathrm{~h}$ increased the apoptotic fraction to $28.2 \pm 2.5 \%$ of sub-G1 cells (Fig. 2A, upper right panel and Fig 2B). A similar effect of $1.0 \mu \mathrm{M}$ Evo and CDK1I on SGC-7901 cells was also observed (Fig. 2A, lower right panel). Quantification indicated that the inclusion of CDK1I significantly increased the apoptosis rate at both 0.5 and $1.0 \mu \mathrm{M}$ Evo compared to Evo treatment alone (Fig. 2B). These results are consistent with the idea that promotion of mitotic slippage by CDK1I enhances apoptosis.

If the induction of apoptosis by Evo was related to mitotic slippage, the promotion or delay of mitotic slippage would be expected to enhance or delay apoptosis, respectively. Therefore, cells were transfected to either express full length or ND cyclinB1 (FL and ND cyclinB1) to modulate cell cycle control. Because cyclinB1 must be degraded before anaphase, the ND cyclinB1 specifically blocked the metaphase-anaphase transition. As previously shown, $36 \mathrm{~h}$ incubation with $1 \mu \mathrm{M}$ Evo caused $52.5 \pm 3.8 \%$ of SGC-7901 cells to die. However, when cells were transfected with ND or FL cyclinB1, subsequent incubation with Evo resulted in a statistically different number $(\mathrm{p}<0.05)$ of sub-G1 cells $(38.2 \pm 4.8 \% ; 51.3 \pm 2.7 \%)$ (Fig. 3). Importantly, transfection of any of the three vectors alone did not affect apoptosis rates. Thus, these results support a model of delayed mitotic slippage as a result of the transient transfection of ND cyclinB1 delaying apoptosis through the blockade of the metaphase-anaphase transition.

Evo affects the endogenous expression of cyclinBl and cyclinE. Cyclin expression was examined in synchronous SGC-7901 cells that were exposed to $1 \mu \mathrm{M}$ Evo for 16, 20, 24 and $36 \mathrm{~h}$. After exposure to Evo for $16 \mathrm{~h}$, there was an increase in cyclinB1 expression (Fig. 4, top panel) as compared to $\beta$-actin (Fig. 4 bottom panel). In contrast, with a 20 -h exposure to Evo, a lower cyclinB1 level was detected compared to $16 \mathrm{~h}$. Additionally, at 24 and $36 \mathrm{~h}$, there was no obvious cyclinB1 expression (Fig. 4 top panel). On the other hand, there was no obvious cyclinE expression after treatment with Evo for $16 \mathrm{~h}$ (Fig. 4, middle panel). However, cyclinE levels increased subsequently as the Evo incubation time increased. These results suggest that a 16-h exposure to Evo arrested cells in mitosis; however, these cells slipped out of mitosis to the G1 phase after experiencing prolonged mitotic arrest.

Evo inhibits colony formation. To examine whether enhanced apoptosis was due to the induction of death after exposure to Evo, cells were grown for 7 additional days and colonies were counted after washout of Evo (Fig. 5). Compared to cells cultured in the absence of Evo, Evo treatment at 0.5 and $1 \mu \mathrm{M}$ inhibited colony formation while $7.5 \mu \mathrm{M}$ CDK1I treatment alone had a modest effect. For cells treated with $0.5 \mu \mathrm{M}$ Evo for $24 \mathrm{~h}$ and co-treated with $7.5 \mu \mathrm{M}$ CDK1I for the last $6 \mathrm{~h}$, the colony inhibition index was $65.3 \pm 6.9 \%$, which was significantly higher than that with $0.5 \mu \mathrm{M}$ Evo alone $(38.0 \pm 3.3 \%)$ or $7.5 \mu \mathrm{M}$ CDK1I alone $(25.1 \pm 4.1 \%)$. Similar effects with $1.0 \mu \mathrm{M}$ Evo treatment were also noticed. These data indicate that mitotic slippage alone was insufficient to induce apoptosis and that mitotic arrest was required for Evo to induce apoptosis. 
A

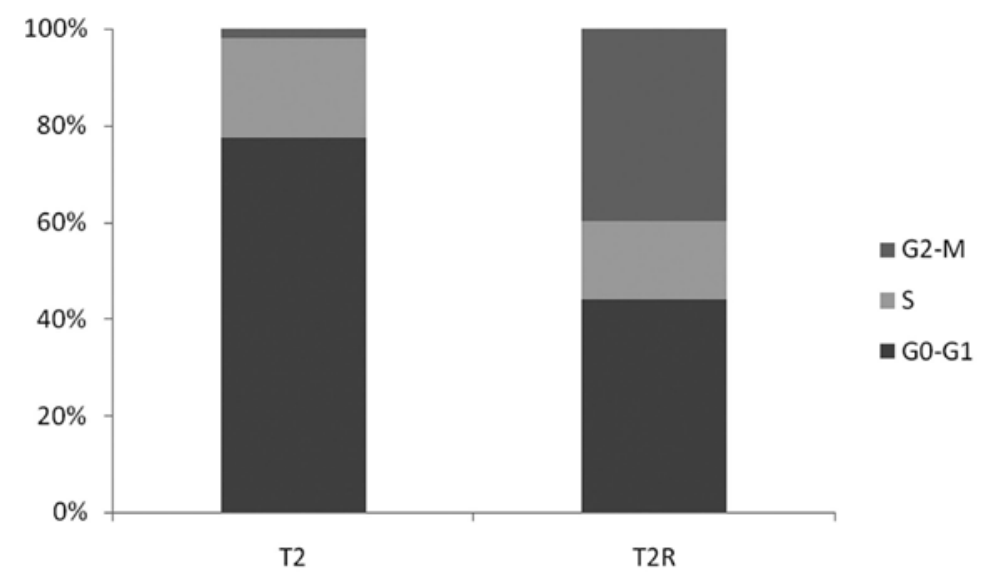

B

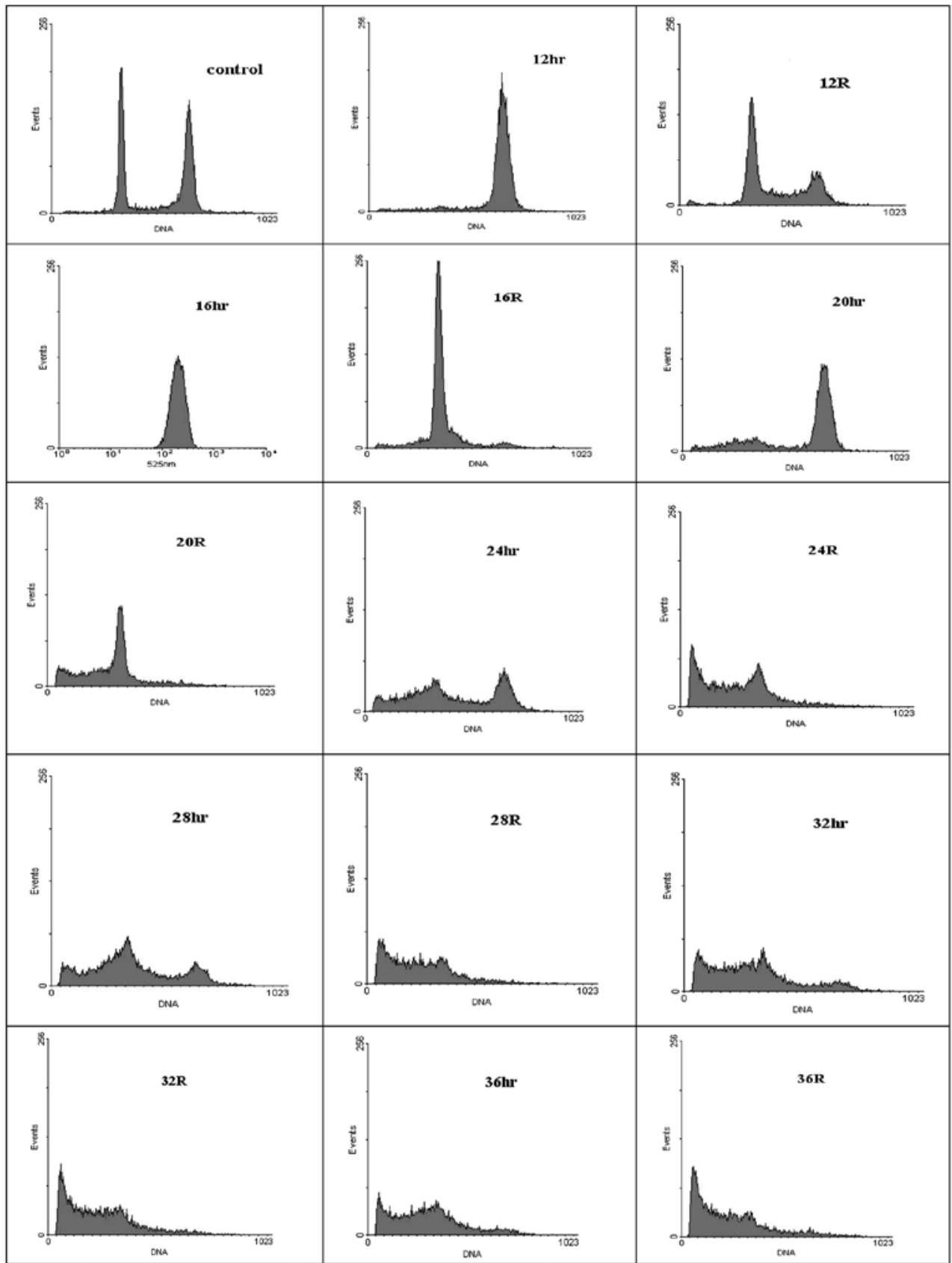

Figure 1A and B. Time-dependent effects of Evo distinguish reversible mitotic arrest from apoptosis. (A) Left, cells were synchronized by TD double block, collected immediately after TD (T2) or after incubation for $12 \mathrm{~h}$ in TD-free medium (T2R) before analyzing for DNA content by FACS. Right, percentage of cells at different phases of the cell cycle was quantified for a representative run. (B) SGC-7901 cells were treated with $1 \mathrm{mM}$ Evo for 12, 16, 20, 24, 28, 32 and $36 \mathrm{~h}$ (non-release group), while the other group was incubated for a further $12 \mathrm{~h}$ in drug-free medium (release group, R), then analyzed with FACS. Control, cells were treated with vehicle only (0.1\% DMSO). 
C

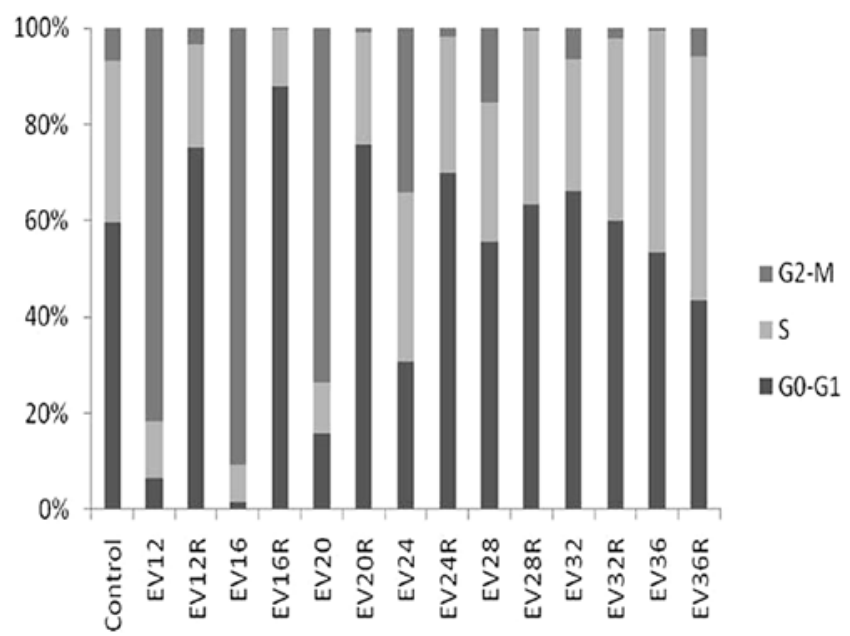

D

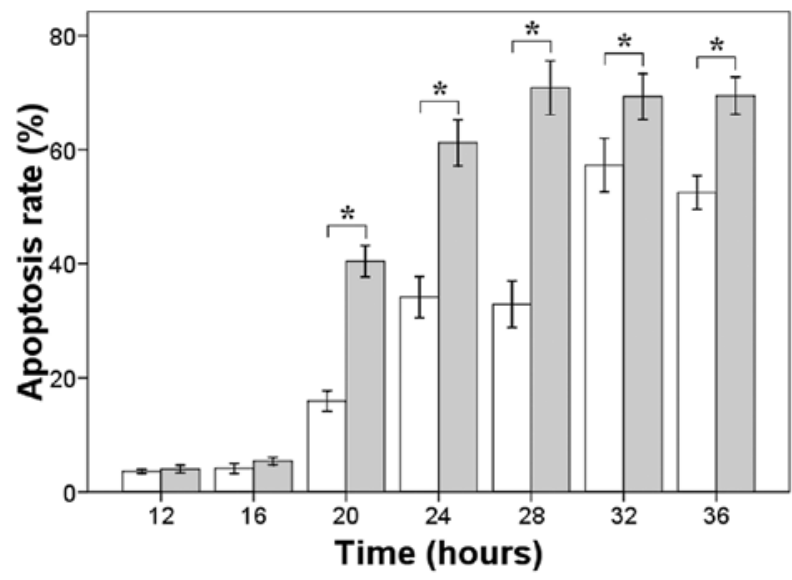

Figure 1C and D. Time-dependent effects of Evo distinguish reversible mitotic arrest from apoptosis. (C) Quantification of the cell cycle distribution of SGC-7901 cells with Evo treatment as shown in B. (D) Apoptosis index for the released group (grey bars) and non-release group (open bars), mean \pm SD, $\mathrm{n}=3$, ${ }^{*} \mathrm{p}<0.05$, compared with release group after Evo treatment for various times.

A

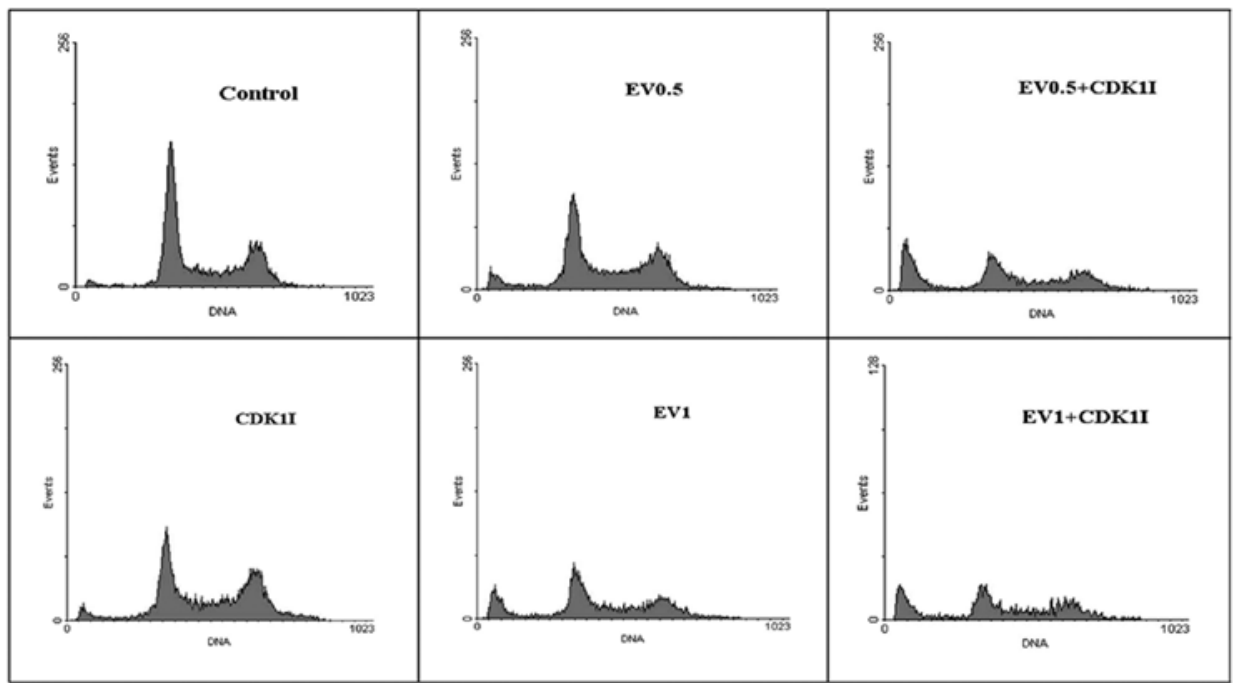

B

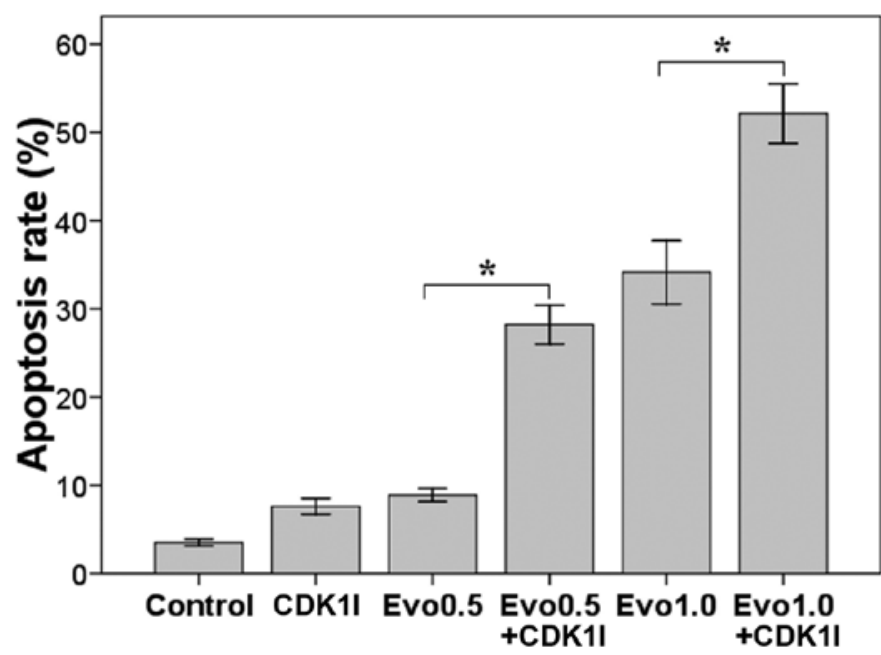

Figure 2. Accelerating mitotic slippage enhances apoptosis. (A) SGC-7901 cells were synchronized and upon release from the block (0 h) cells were treated with $7.5 \mathrm{mM}$ CDK1I, $0.5 \mathrm{mM}$ Evo or $1.0 \mathrm{mM}$ Evo for $24 \mathrm{~h}$ or with $0.5 \mathrm{mM}$ Evo or $1.0 \mathrm{mM}$ Evo for $18 \mathrm{~h}$ and followed by co-incubation with $7.5 \mathrm{mM}$ CDK1I for another $6 \mathrm{~h}$ and analyzed by FACS. (B) Apoptosis index represents mean $\pm \mathrm{SD}, \mathrm{n}=3,{ }^{*} \mathrm{p}<0.05$, compared with co-incubation with $7.5 \mathrm{mM}$ CDK1I. 
A

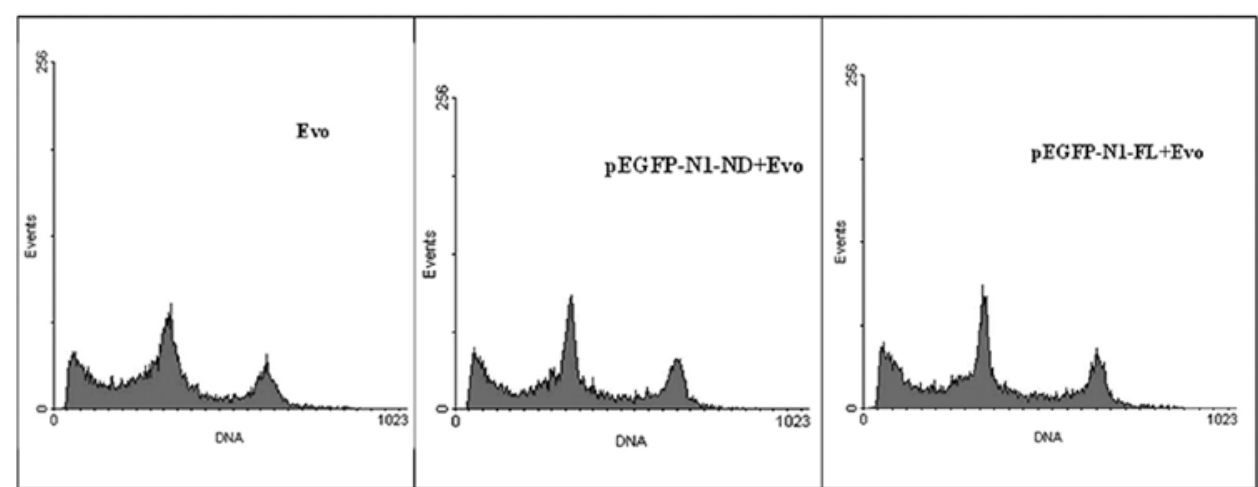

B

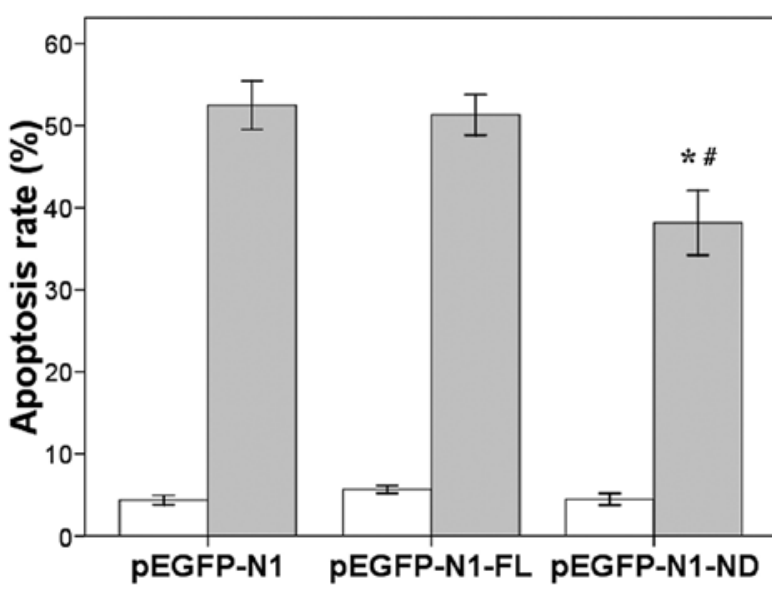

Figure 3. Delaying mitotic slippage delays apoptosis. (A) SGC-7901 cells were transfected with pEGFP-N1-ND cyclinB1 or pEGFP-N1-FL cyclinB1. On release from TD block, cells were then exposed to $1.0 \mathrm{mM}$ Evo for $36 \mathrm{~h}$ and harvested for FACS analysis. (B) Apoptosis index represents mean $\pm \mathrm{SD}$, $\mathrm{n}=3$, "p $<0.05$, compared with pEGFP-N1 + Evo for $36 \mathrm{~h} ;{ }^{*} \mathrm{p}<0.05$ significantly different from pEGFP-N1-FL + Evo.

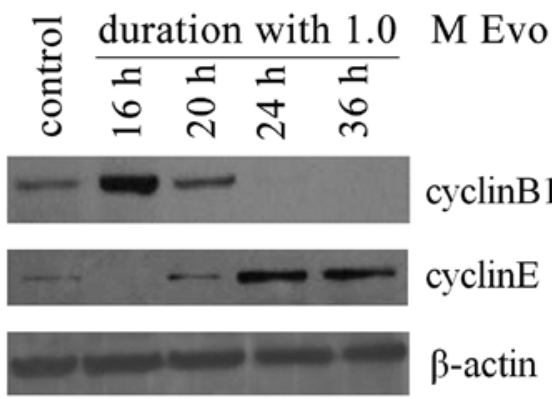

Figure 4. Evo treatment induces expression of cyclinB1 and cyclinE. Expression of cyclinB1 (top panel), cyclinE (middle panel) and $\beta$-actin (bottom panel) was detected by Western blotting of cells treated with (lane 1) vehicle control and $1.0 \mathrm{mM}$ Evo at various time-points as indicated after release from TD block (lanes 2-4).

Evo enhances exogenous gene expression of cyclinB1. Next, we examined the effects of Evo on the exogenous expression of GFP-tagged cyclin B1 using fluorescence intensity as a readout. GFP-tagged cyclinB1 full-length (FL) or non-degradable version (ND) were transfected into SGC-7901 cells while cells transfected with GFP vector alone (pEGFP-N1) served as the negative control. After $36 \mathrm{~h}$ of Evo treatment, $10 \%$

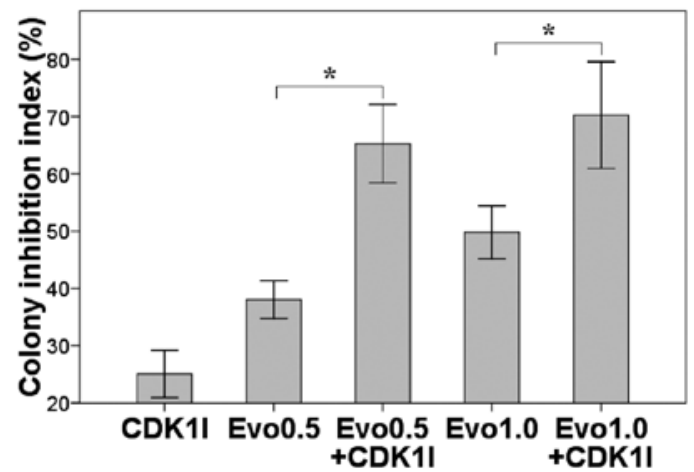

Figure 5. Evo treatment leads to colony inhibition. Upon release from TD block, SGC-7901 cells were treated with vehicle, Evo or $7.5 \mathrm{mM}$ CDK1I for $24 \mathrm{~h}$ or Evo for $24 \mathrm{~h}$ with coincubation of CDK1I for the last $6 \mathrm{~h}$. After washout, cells were cultured for 7 more days before colony counting. Cells were treated with different concentrations of Evo as indicated. Quantification of the colony inhibition index derived from cells represented as the mean $\pm \mathrm{SD}, \mathrm{n}=3,{ }^{*} \mathrm{p}<0.05$ compared with added CDK1I for coincubation.

of cells expressed GFP in cultures transfected with vector alone. The fluorescence was distributed uniformly throughout the cell (cytoplasm and nucleus; Fig. 6A). Then, we analyzed scores for transfections followed by exposure to vehicle or Evo 

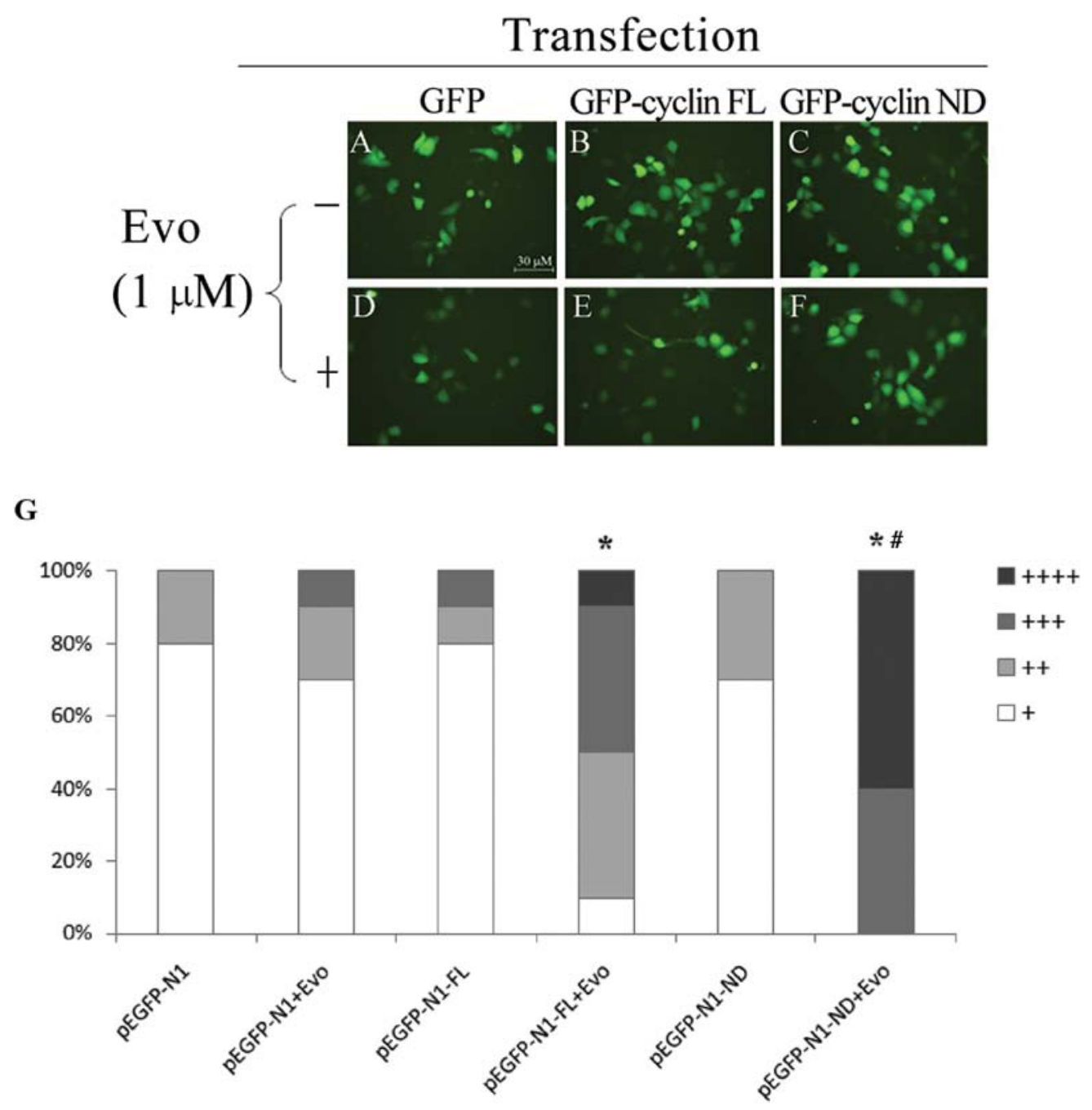

Figure 6. Evo treatment specifically enhances exogenous cyclinB1 expression. Cells were transfected with pEGFP-N1 (A, D), pEGFP-N1-FL cyclinB1 (B, E) or pEGFP-N1-ND cyclinB1 (C,F). Cells were synchronized $24 \mathrm{~h}$ after transfection and upon release from TD block and cells were treated with vehicle alone (top row) or $1.0 \mathrm{mM}$ Evo (lower row) for $36 \mathrm{~h}$ and examined for GFP expression afterwards. (G) Quantification of fluorescence score represented as a percentage, $\mathrm{n}=10 ;{ }^{\mathrm{p}} \mathrm{p}<0.05$ significantly different from pEGFP-N1 + Evo; ${ }^{\#} \mathrm{p}<0.05$ significantly different from pEGFP-N1-FL cyclinB1 + Evo.

for $36 \mathrm{~h}$ (Fig. 6). No significant differences in fluorescence intensity were detected among cell groups transfected but not treated with Evo (Fig. 6A-C and G). On the other hand, with Evo treatment, fluorescence intensity significantly increased $(\mathrm{p}<0.05)$ in the groups transfected with FL and ND cyclinB1 (Fig. 6E-G) compared to cells transfected with vector only (Fig. 6D and G), thereby indicating that Evo treatment was responsible for these differences.

\section{Discussion}

This study demonstrated that Evo markedly inhibited cell growth and was cytotoxic to human gastric cancer cells. For this first time, we demonstrate that short-term Evo treatment $(<20 \mathrm{~h})$ caused a reversible mitotic arrest while sustained mitotic arrest initiated apoptosis, which could be delayed by delaying mitotic slippage with the expression of ND cyclinB1. Furthermore, we demonstrate for the first time that Evo-induced apoptosis can be significantly increased by inhibition of CDK1, which is a key regulator of the G2/M phase transition. Thus, the induction of apoptosis by Evo required both activation of mitotic arrest and subsequent mitotic slippage. These findings not only confirm previous studies demonstrating that Evo is an anti-tumor agent that affects the mitotic phase of the cell cycle $(12,13)$, but they also give new insights into the apoptotic effects of this compound, whereby the inappropriate progression to interphase eventually leads to cell death. Moreover, these findings may be generalizable to other types of tumor cells because the apoptotic effects of Evo have been demonstrated in multiple cells types $(10,25,26)$. Among these various types of tumor cells, Evo has been suggested to mediate its effects by arresting the cell cycle at the M phase, $(10,25)$ with the only exception being a recent study that used a significantly higher concentration of Evo (7.5-60 vs. 0.5-1.0 $\mu \mathrm{M}$ used in most studies) and showed $S$ phase arrest (26).

The idea of cell cycle checkpoints was developed by Hartwell and Weinert (15), which are now considered an essential mechanism in regulating cell cycle progression. When cells enter the mitotic phase, the spindle assembly checkpoint (SAC) monitors the mitotic phase to ensure the proper distribution of genetic 
material. The SAC can inhibit the activity of the anaphasepromoting complex (APC) in order to delay cells from slipping out of the mitotic phase (27). Any factor impacting CDK1 and/ or cyclinB1 will activate SAC, inactivate APC and arrest cells at the $\mathrm{G} 2 / \mathrm{M}$ phase. Generally, when the cells satisfy the conditions of SAC, the arresting signals are relieved; otherwise, cells are maintained in the mitotic phase.

However, cells can also enter the mitotic phase without meeting the conditions of SAC; although the mechanism remains unclear. The phenomenon we term 'mitotic slippage' means that a cell has overcome the SAC with a partially damaged spindle apparatus (28). Cells, by some means, inactivate cyclinB/CDK1, thereby allowing them to transition out of mitosis under conditions that would typically fail to meet SAC standards. Kinesin spindle protein (KSP) is a microtubule motor protein and KSP inhibitor-induced apoptosis requires activation of mitotic arrest and mitotic slippage. Notably, these two events are indispensable for the cell to undergo mitotic slippage $(16,20,29)$.

According to FACS analysis of the cell cycle distribution and apoptosis index, many cells were arrested at the G2/M phase after exposure to Evo for $12 \mathrm{~h}$. Furthermore, mitotic arrest needed to be maintained for 4-8 h before these cells entered an irreversible apoptotic pathway. Exposure to Evo for $<16-20 \mathrm{~h}$, eventually allowed cells to resume a normal cell cycle. This finding adds an important facet to the time course for Evo treatment and this is the first study to demonstrate that a tumor cell line can recover from brief $(<16-20 \mathrm{~h})$ Evo treatment.

Expression of cyclinB1 is a marker for the G2/M phase and cyclinE is expressed in the G1 phase (30). Western blot analysis indicated that cyclinB1 increased after exposure to Evo for $16 \mathrm{~h}$, but decreased with longer exposure and became undetectable at $36 \mathrm{~h}$ (Fig. 4), thereby indicating that these cells had progressed out of the $M$ phase by $36 \mathrm{~h}$. In contrast, cyclinE began to be expressed after exposure for $24 \mathrm{~h}$ and peaked after $36 \mathrm{~h}$, which indicated a transition to G1. Together these findings are consistent with Evo treated cells undergoing mitotic slippage.

If the induction of apoptosis by Evo is related to mitotic slippage, promotion or delay of mitotic slippage would be expected to enhance or delay apoptosis, respectively. CDK1I integrates with CDK1 directly or with a cyclinB1-CDK1 complex to regulate the cell cycle, thereby making cells move out of the mitotic phase and into the G1 phase. Although the results of flow cytometry and the colony survival assay were different, they both showed the induction of apoptosis by Evo, and that promotion of mitotic slippage enhanced apoptosis. Exposure to CDK1I indicated that mitotic slippage alone without activation of the spindle checkpoint was insufficient to induce apoptosis, whereas promotion of mitotic slippage by CDK1 inhibition greatly enhanced apoptosis.

Typical cyclical changes of cyclin family members are associated with their degradation box. Non-degradable cyclinB1 (ND cyclinB1) has the N-terminus degradation box removed and has been previously used to study cell cycle regulation (31). In this study, we expressed ND cyclinB1 and FL cyclinB1 target genes in SGC-7901 cells to explore the relationship between Evo-induced apoptosis and cell cycle regulation. Interestingly, overexpression of the ND cyclinB1 but not the full-length cyclinB1 significantly reduced Evo-induced apoptosis rates
(Fig. 3), which most likely reflected mitotic arrest caused by the sustained expression of this cyclin due to the absence of its degradation domain. Furthermore, higher expression was observed in cells transfected with these cyclinB1 constructs (Fig. 6). Thus, these data indicate that delaying mitotic slippage after Evo exposure can delay apoptosis.

It has been reported that Evo-induced apoptosis in a timeand dose-dependent manner $(25,26,32)$; however, our study used a much lower dose $(1 \mu \mathrm{M})$ that may be more clinically relevant. In fact, the inhibitory effect of Evo on tumor cells at low concentrations is significant $(6,12)$. Previous studies have demonstrated that this effect of Evo may involve the up-regulation of Bax, down-regulation or phosphorylation of $\mathrm{Bcl}-2$ and/or the activation of certain caspases $(5,12,26,33)$. It has also been demonstrated that one of the mechanisms underlying the apoptotic effect of Evo is mediated by inhibiting NF- $\kappa \mathrm{B}$ activation $(34,35)$. Hence, Evo appears to affect the activation of several apoptotic pathways that contribute to its anti-proliferative effects on tumor cells. Recently, Evo was shown to directly bind to DNA and form an inhibitory complex with topoisomerase I, which may contribute to its anti-tumor effects (36).

In summary, the present investigation suggests that evodiamine-induced cell apoptosis may be related to activating mitotic arrest and mitotic slippage, which may contribute to the mechanism underlying the actions of this natural compound for the treatment and prevention of cancer.

\section{Acknowledgements}

This study was supported by a research grant from the National Natural Science Foundation of China (30672747).

\section{References}

1. Wu CL, Hung CR, Chang FY, Lin LC, Pau KY and Wang PS: Effects of evodiamine on gastrointestinal motility in male rats. Eur J Pharmacol 457: 169-176, 2002.

2. Chiou WF, Chou CJ, Shum AYC and Chen CF: The vasorelaxant effect of evodiamine in rat isolated mesenteric arteries: mode of action. Eur J Pharmacol 215: 277-283, 1992.

3. Rang WQ, Du YH, Hu CP, et al: Protective effects of evodiamine on myocardial ischemia-reperfusion injury in rats. Planta Med 70: 1140-1143, 2004.

4. Tang W and Eisenbrand G (eds): Evodia rutaecarpa (Juss) Benth. In: Chinese Drugs of Plant Origin. Springer-Verlag, Berlin, pp509-514, 1992

5. Fei XF, Wang BX, Li TJ, et al: Evodiamine, a constituent of Evodiae Fructus, induces anti-proliferating effects in tumor cells. Cancer Sci 94: 92-98, 2003.

6. Kan SF, Yu CH, Pu HF, Hsu JM, Chen MJ and Wang PS: Antiproliferative effects of evodiamine on human prostate cancer cell lines DU145 and PC3. J Cell Biochem 101: 44-56, 2007.

7. Yang ZG, Chen AQ and Liu B: Antiproliferation and apoptosis induced by evodiamine in human colorectal carcinoma cells (COLO-205). Chem Biodivers 6: 924-933, 2009.

8. Chen MC, Yu CH, Wang SW, et al: Anti-proliferative effects of evodiamine on human thyroid cancer cell line ARO. J Cell Biochem 110: 1495-1503, 2010.

9. Hsia SM, Su JC, Wang KL and Wang PS: Effects of evodiamine on the expression and secretion of vascular endothelial growth factor in human breast cancer cells. Clin Mol Med 1: 8-13, 2010.

10. Jiang $\mathrm{J}$ and $\mathrm{Hu} \mathrm{C}$ : Evodiamine: a novel anti-cancer alkaloid from Evodia rutaecarpa. Molecules 14: 1852-1859, 2009.

11. Li-Weber M: Targeting apoptosis pathways in cancer by Chinese medicine. Cancer Lett: Aug 2, 2010 (Epub ahead of print).

12. Huang YC, Guh JH and Teng CM: Induction of mitotic arrest and apoptosis by evodiamine in human leukemic T-lymphocytes. Life Sci 75: 35-49, 2004. 
13. Huang DM, Guh JH, Huang YT, Chueh SC, Chiang PC and Teng CM: Induction of mitotic arrest and apoptosis in human prostate cancer PC-3 cells by evodiamine. J Urol 173: 256-261, 2005.

14. King KL and Cidlowski JA: Cell cycle and apoptosis: common pathways to life and death. Cell Biochem 58: 175-180, 1995.

15. Hartwell LH and Weinert TA: Checkpoints: controls that ensure the order of cell cycle events. Science 246: 629-634, 1989.

16. Tao W, South VJ, Zhang Y, et al: Induction of apoptosis by an inhibitor of the mitotic kinesin KSP requires both activation of the spindle assembly checkpoint and mitotic slippage. Cancer Cell 8: 49-59, 2005.

17. Vogel C, Kienitz A, Müller R and Bastians H: The mitotic spindle checkpoint is a critical determinant for topoisomerase-based chemotherapy. J Biol Chem 280: 4025-4028, 2005.

18. Stolz A, Vogel C, Schneider V, et al: Pharmacologic abrogation of the mitotic spindle checkpoint by an indolocarbazole discovered by cellular screening efficiently kills cancer cells. Cancer Res 69: 3874-3883, 2009.

19. Jordan MA and Wilson L: Microtubules as a target for anticancer drugs. Nat Rev Cancer 4: 253-265, 2004.

20. Sudo T, Nitta M, Saya H and Ueno NT: Dependence of paclitaxel sensitivity on a functional spindle assembly checkpoint. Cancer Res 64: 2502-2508, 2004.

21. Shi J, Orth JD and Mitchison T: Cell type variation in responses to antimitotic drugs that target microtubules and kinesin-5. Cancer Res 68: 3269-3276, 2008.

22. Lowe SW and Lin AW: Apoptosis in cancer. Carcinogenesis 21: 485-495, 2000

23. Whitfield ML, Sherlock G, Saldanha AJ, et al: Identification of genes periodically expressed in the human cell cycle and their expression in tumors. Mol Biol Cell 6: 1977-2000, 2002.

24. Suzuki K, Hino M, Kutsuna H, Hato F, Sakamoto $C$ and Takahashi T: Selective activation of p38 mitogen-activated protein kinase cascade in human neutrophils stimulated by IL-1ß. J Immunol 167: 5940-5947, 2001.

25. Yang J, Wu LJ, Tashino S, Onodera S and Ikejima T: Reactive oxygen species and nitric oxide regulate mitochondria-dependent apoptosis and autophagy in evodiamine-treated human cervix carcinoma HeLa cells. Free Radic Res 42: 492-504, 2008.
26. Zhang $C$, Fan $X, X u X$, Yang $X$, Wang $X$ and Liang HP Evodiamine induces caspase-dependent apoptosis and $\mathrm{S}$ phase arrest in human colon lovo cells. Anticancer Drugs 21: 766-776, 2010.

27. Musacchio A and Salmon ED: The spindle-assembly checkpoint in space and time. Nat Rev Mol Cell Biol 8: 379-393, 2007.

28. Brito DA, Yang Z and Rieder CL: Microtubules do not promote mitotic slippage when the spindle assembly checkpoint cannot be satisfied. J Cell Biol 182: 623-629, 2008.

29. Chen JG, Yang CPH, Cammer M and Horwitz SB: Gene expression and mitotic exit induced by microtubule-stabilizing drugs. Cancer Res 63: 7891-7899, 2003.

30. Darzynkiewicz Z, Gong J, Juan G, Ardelt B and Traganos F: Cytometry of cyclin proteins. Cytometry 25: 1-13, 1996.

31. Wolf F, Wandke C, Isenberg N and Geley S: Dose-dependent effects of stable cyclin B1 on progression through mitosis in human cells. EMBO J 25: 2802-2813, 2006.

32. Huang H, Zhang Y, Liu X, Li Z, Xu W, He S, Huang Y and Zhang H: Acid sphingomyelinase contributes to Evodiamineinduced apoptosis in human gastric cancer SGC-7901 cells. DNA Cell Biol 30: 407-412, 2011.

33. Zhang $\mathrm{Y}, \mathrm{Wu} \mathrm{LJ}$, Tashiro S, Onodera $\mathrm{S}$ and Ikejima T: Intracellular regulation of evodiamine-induced A375-S2 cell death. Biol Pharm Bull 26: 1543-1547, 2003.

34. Takada Y, Kobayashi Y and Aggarwal BB: Evodiamine abolishes constitutive and inducible NF-kappaB activation by inhibiting IkappaBalpha kinase activation, thereby suppressing NF-kappaB-regulated antiapoptotic and metastatic gene expression, up-regulating apoptosis, and inhibiting invasion. J Biol Chem 280: 17203-17212, 2005.

35. Wang C, Li S and Wang MW: Evodiamine-induced human melanoma A375-S2 cell death was mediated by PI3K/Akt/caspase and Fas-L/NF-kappaB signaling pathways and augmented by ubiquitin-proteasome inhibition. Toxicol In Vitro 24: 898-904, 2010.

36. Chan AL, Chang WS, Chen LM, Lee CM, Chen CE, Lin CM and Hwang JL: Evodiamine stabilizes topoisomerase I-DNA cleavable complex to inhibit topoisomerase I activity. Molecules 14: $1342-1352,2009$ 